\title{
Acute corneal hydrops in keratoconus: a national prospective study of incidence and management
}

A Barsam², H Petrushkin², N Brennan³ ${ }^{3}$ C Bunce², W Xing ${ }^{2}$, B Foot $^{4}$ and S Tuft ${ }^{2}$

\begin{abstract}
Purpose To determine incidence and management of acute corneal hydrops in the UK.

Methods We used the BOSU report card system to survey cases of acute corneal hydrops in patients with keratoconus that occurred in the UK between November 2009 and December 2010. Ophthalmologists who reported a case were sent an initial questionnaire, with a follow-up questionnaire after 6 months. We collected information on the demographics, complications, changes in visual acuity, and management. The 2011 National Census was used as a source for population and ethnicity in the UK.

Results There were 73 incident cases of acute corneal hydrops, with a response to the initial questionnaire for $64(88 \%)$ patients and follow-up data at 6 months for $57(78 \%)$ patients. For the 64 confirmed cases the median (interquartile range) age of onset was $31.9(23.2,41.3)$ years and $48(75 \%)$ of the cases occurred in males. A total of $42(66 \%)$ patients were white, $14(22 \%)$ were South Asian, and 7 (11\%) were black. The proportion of South Asian and black patients with acute corneal hydrops was significantly higher than in the general population $(P<0.001)$. The minimum estimated annual incidence of acute corneal hydrops in patients with keratoconus was estimated to be $1.43(1.10,1.83)$ per 1000 . At 6 months following acute corneal hydrops a decision to proceed with keratoplasty had been made for $12(20.3 \%)$ patients.

Conclusions This is the first populationbased estimate of the incidence of acute corneal hydrops in keratoconus.
\end{abstract}

Eye (2015) 29, 469-474; doi:10.1038/eye.2014.333; published online 16 January 2015

\section{Introduction}

Keratoconus is associated with biomechanical abnormalities of the cornea that leads to secondary thinning and ectasia. Distortion of the cornea results in keratometric myopia and irregular astigmatism that can severely affect vision. The aetiology is unknown and corneal ectasia may represent the final common pathway for a variety of separate disease processes. ${ }^{1}$ In mild cases visual correction may be achieved with glasses, but as corneal distortion progresses rigid contact lenses are often required. In hospital-based populations $\sim 10 \%$ of patients require a keratoplasty to improve their vision within 10 years of diagnosis, ${ }^{2}$ and in developed countries keratoconus is the most common indication for keratoplasty. ${ }^{3}$ It normally presents in early adulthood, it is more common in males, and typically progresses until the fourth decade of life when the disease usually stabilises. It affects patients of all ethnicities but with a higher prevalence in South Asian than white patients. ${ }^{4}$ There is a very wide range in the reported incidence of keratoconus of between 1.3 to 25 new cases per 100000 per year, ${ }^{5-7}$ with estimates of prevalence of between 0.2 and 2300 individuals per 100000 individuals, with differences determined by the inclusion and the diagnostic criteria used for case ascertainment, and the age or ethnicity of the population studied. $2,4,5,7-10$

Acute corneal hydrops is a complication of keratoconus that develops when there is a split in the Descemet layer and endothelium that allows aqueous to enter the cornea with gross
${ }^{1}$ Department of Ophthalmology, Luton and Dunstable University Hospital, Luton, UK

${ }^{2}$ Corneal Service, Moorfields Eye Hospital NHS Foundation Trust, London, UK

${ }^{3}$ Department of Ophthalmology, Hillingdon Hospital, London, UK

${ }^{4}$ British Ophthalmic Surveillance Unit, The Royal College of Ophthalmologists, London, UK

Correspondence: $S$ Tuft, Moorfields Eye Hospital NHS Foundation Trust, 162 City Road, London EC1V 2PD, UK Tel: +44 (0)20 7566 2375; Fax: +44 (0)20 75662019 . E-mail: s.tuft@ucl.ac.uk

Received: 6 July 2014 Accepted in revised form: 20 November 2014 Published online: 16 January 2015 
oedema of the overlying stroma and epithelium. ${ }^{11}$ It has also been described as a complication of pellucid marginal degeneration and keratoglobus. ${ }^{12,13}$ Acute corneal hydrops has been associated with rapidly progressive keratoconus with an onset within the first few years of diagnosis. In the majority of patients an acute corneal hydrops resolves uneventfully over 2-4 months, ${ }^{14}$ although vascularisation, ${ }^{15}$ infection, ${ }^{16}$ and perforation ${ }^{16}$ are potential complications. The presence of a residual corneal scar after hydrops can reduce vision to an extent that a keratoplasty is required to improve vision. A prior history of acute corneal hydrops has been reported to be a risk factor for allograft graft rejection. ${ }^{2}$

Although the prevalence of an episode of corneal hydrops in clinic-based patients with keratoconus has been estimated to be between $2.6^{17}$ and $2.8 \%,{ }^{18}$ to the best of our knowledge there are no published estimates of the incidence of acute corneal hydrops in keratoconus. ${ }^{19} \mathrm{We}$ therefore conducted a survey of all ophthalmologists in the UK during a 12-month interval to identify new cases of acute corneal hydrops. We used two questionnaires to collect data on the epidemiology of acute corneal hydrops and the management strategies that were employed. We collated this information with national census data to derive an incidence figure.

\section{Materials and methods}

We used the British Ophthalmological Surveillance Unit (BOSU) card reporting system to prospectively identify new cases of acute corneal hydrops that occurred in patients in the UK between November 2009 and December 2010. A case of acute corneal hydrops was defined as the sudden onset of bullous corneal stromal oedema in a patient with keratoconus with a confirmed break in the Descemet layer. The diagnosis of keratoconus was based on agreed criteria. ${ }^{20}$ Patients were included if they were normally residents in the UK, and there were no age restrictions. At the end of each calendar month a BOSU report card was sent to all consultant and associate specialist ophthalmologists in the UK. Recipients were asked to indicate all new cases of acute corneal hydrops or to confirm that they had no new cases to report. When a case of acute corneal hydrops was reported the ophthalmologist was sent an initial questionnaire requesting clinical and demographic details including age, gender, racial group, best corrected visual acuity (BCVA) before the onset of acute corneal hydrops, and management. Ethnicity was grouped as white, South Asian, South East Asian, or black, with mixed ethnicity grouped as mixed or other (Table 1). A second follow-up questionnaire at 6 months requested details of any complications, the last recorded corrected visual acuity (VA) after the onset of hydrops, and any surgical intervention or planned surgical intervention. If vision was recorded as Snellen acuity it was converted to LogMAR equivalents. The study had research ethical approval (09/H0706/5).

Because the number of patients with keratoconus in the UK is unknown, we based our prevalence estimate on data from Denmark published by Nielsen et al. ${ }^{7}$ This estimate was based on the number of patients with the ICD10 classification for keratoconus (DH18.6) in the population of susceptible age ( $>15$ years of age) for the years 1995-2005. The population and ethnicity of the UK was derived from the UK 2011 National Census, which are the data closest to collection interval of the study. ${ }^{21-23}$ Descriptive analysis was used to report complications and treatment strategies. Summary measures were presented as mean and standard deviation for continuous (approximate) normally distributed variables, median and interquartile ranges (IQR) for non-normally distributed variables, and frequencies and percentages for categorical variables. Binomial probability tests were used to assess whether there was evidence that the proportion of each ethnic group was greater than would be expected from census figures. $\chi^{2}$ tests were used to assess whether there was evidence of a greater proportion of males than might be attributed to chance. All analyses were conducted using STATA Statistics/Data Analysis version 12.

\section{Results}

A total of 73 cases of acute corneal hydrops were reported during the study period. The response rate for

Table 1 Ethnic distribution of 64 patients with acute corneal hydrops reported in the 12-month interval November 2009 to December 2010 compared with the population of England and Wales, Scotland, and Northern Ireland in the UK census 201122,23,36

\begin{tabular}{lcccc}
\hline Ethnicity & Study, No. (\%) & UK Census data 2011, No. (\%) & Incidence (per 10 $\left.{ }^{5}\right)$ & 95 \% confidence interval \\
\hline White & $42(65.6)$ & $55088140(87.2)$ & 0.07 & $0.055,0.103$ \\
Asian $^{\mathrm{a}}$ & $14(21.9)$ & $4359198(6.9)$ & 0.32 & $0.176,0.539$ \\
Black & $7(10.9)$ & $1883759(3.0)$ & 0.37 & $0.149,0.766$ \\
Mixed or other & $1(1.6)$ & $1824725(2.9)$ & 0.05 & $0.001,0.305$ \\
\hline
\end{tabular}

Abbreviation: No., number.

${ }^{a}$ All cases were South Asian (7 Indian, 2 Bangladeshi, and 5 Pakistani). 
the initial questionnaire was $64(88 \%)$ patients and complete data for the 6-month follow-up was $57(78 \%)$ patients. The right eye was affected in 37 (57.8\%) cases and $48(75 \%)$ were male. The observed male to female ratio was statistically significantly different to a $1: 1$ ratio $(P<0.001)$. The median (IQR) interval from diagnosis of keratoconus to the development of acute corneal hydrops in the 56 subjects for whom these dates were available was $8.7(1.1,15.7)$ years. The median (IQR) age at diagnosis of hydrops was $31.9(23.2,41.3)$ years. In ten patients the diagnosis of keratoconus was first made at presentation with an acute corneal hydrops. None of the patients was reported to have pellucid marginal degeneration or keratoglobus. The ethnicity of patients is presented in Table 1 . There was a significantly higher proportion of patients reported as South Asian or black than in the general population for the 2011 UK census. $^{22,23}$ There were no cases of acute corneal hydrops in patients reported as South East Asian. The estimated incidence of hydrops in white subjects was 0.07 per 100000 compared with an estimated incidence of 0.32 per 100000 for South Asian subjects and 0.37 for black subjects, so that South Asians and black subjects appears to be at a much greater risk of hydrops than white subjects (rate ratio $4.21(2.21,7.87)$ and $4.87(1.85,10.95)$, respectively). Nine patients had a history of a previous hydrops and in six of these patients the recurrence was in the same eye; six of the nine patients in whom a recurrence occurred were non-white.

Of the 64 patients providing data, $29(46.8 \%)$ were treated with a topical corticosteroid and $32(50 \%)$ were treated with hypertonic (5\%) saline. Four patients received oral antibiotics (no explanation given). Six (10.2\%) eyes developed significant corneal neovascularisation after their hydrops, and two of these patients were atopic. Two eyes (3.4\%) developed a corneal perforation, and in one eye $(1.5 \%)$ this was complicated by a sterile endophthalmitis. Seven (11.9\%) eyes had received a penetrating keratoplasty (PK) within 6 months of their hydrops and a further four (6.8\%) eyes had been scheduled for keratoplasty. No patients were reported to have had deep anterior lamellar keratoplasty or anterior chamber gas injection. The median LogMar BCVA before the onset of hydrops (38 eyes) was 0.6 (IQR 0.3-1), the uncorrected VA at the time of hydrops (54 eyes) was 1.6 (IQR 1.5-1.9) and the BCVA reported at 6 months after hydrops (47 eyes) was 1.3 (IQR 0.6-1.6; Figure 1).

The population of the UK at the time of the 2011 census was 63.2 million and we defined the population at risk of keratoconus as $>15$ years of age (52.1 million persons). ${ }^{21}$ We assumed that the prevalence of keratoconus in the UK was the same as in Denmark and used a figure of $1: 1163 .^{7}$ From this we estimated that there were

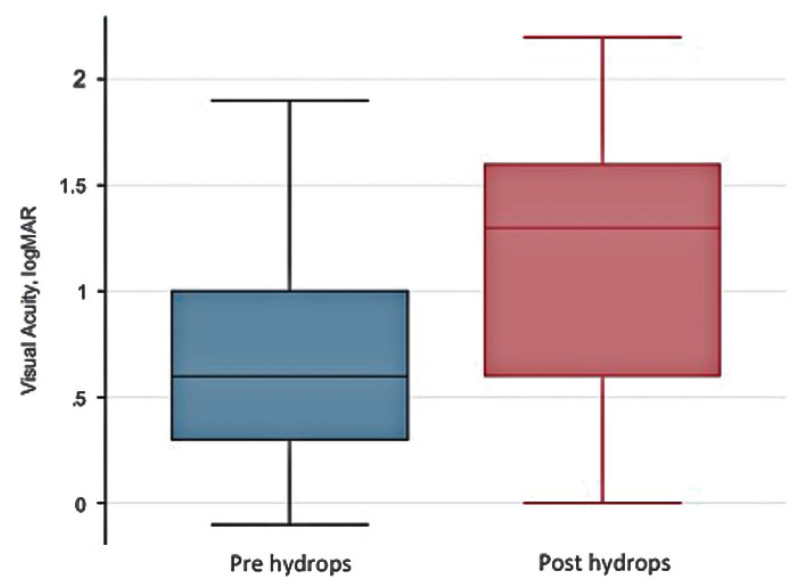

Figure 1 Box plots demonstrating change in visual acuity for 33 eyes with data from before and after the onset of acute corneal hydrops. Vision after hydrops was statistically significantly worse than vision prior to hydrops. ( $P=0.017$, signed-rank test).

44712 people $>15$ years of age living in the UK with keratoconus in contact with hospital services. This gives a figure for the minimum estimated annual incidence of acute corneal hydrops in the population at risk of keratoconus of $1.43(1.10,1.83)$ per 1000 (using the figure of 64 confirmed cases) or $1.63(1.27,2.05)$ per 1000 (using the reported 73 cases).

\section{Discussion}

Acute corneal hydrops is the sudden onset of gross corneal oedema in an eye with keratoconus that typically causes a marked reduction of vision. The associated blur and discomfort can adversely affect the ability to work and quality of life. Although the hydrops normally resolves over a period of 3-4 months residual corneal scar can still reduce vision, when compared with the corrected acuity before the onset of hydrops. Previously published figures for the prevalence of acute corneal hydrops have been derived from selected populations and thus probably overestimate the risk. This prospective population-based study has allowed us to estimate the minimum incidence of acute corneal hydrops in the UK in hospital-registered patients with keratoconus. Our upper estimate of $1.63(1.27,2.05)$ per 1000 patients with keratoconus compares with previously published prevalence estimates of $2.6^{17}$ to $2.8 \%^{18}$ based on retrospective data gathered in specialist referral clinics.

It has been reported previously that South Asian or black patients are at especial risk of developing keratoconus, 4,24 and we have shown that South Asian or black ethnicity is also a risk factor for the development of acute corneal hydrops. It has been estimated that in the UK there is a 4.0- to 7.5-fold increase in the prevalence of keratoconus in South Asians compared with white 
patients, with a younger age at presentation. ${ }^{4,6}$ In a retrospective case series of 101 episodes of corneal hydrops in 101 patients reported from New Zealand, individuals of Pacific, but not Maori, ethnicity were reported to have a higher risk of hydrops compared with White Europeans. ${ }^{19}$ Although we observed that hydrops was also more common in South Asian and black patients than white patients we cannot say whether the proportions of patients with keratoconus who progress to corneal hydrops differs between groups. The relevance of the influence of race is that our results will only apply to populations with a similar racial mix, and may be expected to change over time if there is migration. Our study confirmed previous observations that acute corneal hydrops is more common in males with a ratio of $3: 1$ compared with previous estimates of $1.2: 1$ to $2.9: 1.17,19,25$ The median age of onset in the present study was 31.9 years compared with previous reports of 24.6-39.3 years. ${ }^{17,19,25}$ The observation that keratoconus may first be diagnosed at the presentation of an acute corneal hydrops has been reported previously. ${ }^{19}$

A wide range of treatments have been recommended for acute corneal hydrops, although none have been validated in a controlled trial. ${ }^{26,27}$ All patients in this study were treated with a topical antibiotic, lubricants and cycloplegia, whereas a proportion of patients also received topical hypertonic saline or corticosteroid. It is unlikely that any medical therapy hastens the resolution of the hydrops. ${ }^{25}$ Corneal hydrops can stimulate corneal neovascularisation, particularly if the hydrops extends to the limbus with large stromal clefts, or if there is inflammation such as severe coexisting allergic eye disease, and topical corticosteroid has been recommended to reduce this risk. ${ }^{15}$ Other secondary complications such as microbial infection or corneal perforation following acute corneal hydrops are uncommon and may be associated with coexisting chronic allergic conjunctivitis or a return to contact lenses. ${ }^{17,25}$ The utility of a prophylactic antibiotic following an acute corneal hydrops is unproven.

Although the majority of cases of acute corneal hydrops resolve within 4 months, ${ }^{25}$ resolution may be slower in younger patients, or if there is a large Descemet layer break and large intrastromal clefts. ${ }^{28,29}$ Extensive corneal involvement has been associated with slower resolution of the hydrops and a poor final acuity. ${ }^{25}$ It has been reported that the resolution of a small hydrops can be accelerated by injection of gas (eg, air or isovolumetric SF6 or C3F8), but there are risks of pupil block and the final visual outcome is not improved. ${ }^{11,30}$ No patients in our series were reported to have had a gas injection. It is also usual to await resolution of hydrops to assess the corrected acuity before performing keratoplasty because secondary scarring may flatten the cornea, which can improve rigid contact lens fit with useful vision if there is a scar off the visual axis. ${ }^{17,25}$ However, none of the patients in our study had an improved visual acuity as a result of their acute corneal hydrops. The deterioration in vision following a hydrops may encourage patients to have a keratoplasty, and previous studies have reported that the proportion of patients receiving keratoplasty following hydrops is between $18-67 \% .{ }^{17,19,25}$ A previous study from a contact lens department in the UK reported that $59.2 \%$ of eyes with hydrops had a keratoplasty compared with $13.1 \%$ in eyes without hydrops. ${ }^{17}$ In contrast, a study from New Zealand reported that patients with hydrops did not have an increase in the rate of keratoplasty compared with age-matched controls with keratoconus, ${ }^{19}$ although the authors noted that the proportion of keratoplasties performed for keratoconus in New Zealand (45.6\%) is higher than in other published series $^{31}$ All patients in the current study had PK.

Although the risk of rejection may be reduced following deep lamellar keratoplasty compared with PK the visual outcome may be inferior because of incomplete scar removal. ${ }^{32,33}$

The limitations of our study are the relatively short follow-up period and the reliance on BOSU as the sole source for reporting new cases of acute corneal hydrops. The mean-card return rate to the BOSU for the 12-month study period was $76 \%$, which provides an indicator of good compliance from reporting ophthalmologists, but the BOSU reporting scheme is dependent on voluntary reporting and systematic under-reporting by individuals may have biased the data. The values for prevalence are based on the hospital records and they are likely to be an overestimate compared with the population as a whole. We do not have any information on the number of cases of keratoconus managed by optometrists in the community. Similarly, the number of cases of acute corneal hydrops, if any, that are managed outside the hospital system is unknown. The 6-month follow-up is also insufficient to identify the total number of cases proceeding to surgery; the mean interval to keratoplasty following an acute hydrops has been reported to be 1.3 years for right eye and 2.3 years for left eye. ${ }^{17}$ It has also been reported that the risk of allograft rejection after PK is increased if there is a prior hydrops, ${ }^{17,34}$ but, again, the follow-up in this study was too short to permit us to comment on this. Although acute corneal hydrops may be more common in sporadic cases of keratoconus that in patients with a family history, the proportion of individuals in this study with inherited keratoconus was unknown. We used an estimate of the prevalence of keratoconus in Denmark to calculate a figure for the prevalence of keratoconus in the UK. However, the proportion of the Danish population that is of South Asian decent is $<0.5 \%$ compared with $6.9 \%$ in the UK. ${ }^{35}$ 
Because keratoconus is more prevalent in South Asians our figure for the number of cases of keratoconus in the UK is therefore an underestimate. Finally, keratoconus can occur before the age of 15 years, particularly in South Asian and black patients, and therefore the estimate of the population at risk may have been inaccurate.

In conclusion, we report estimates for the incidence of acute corneal hydrops in the UK population. The results confirm the strong effect of ethnicity and provide data that is useful for patient counselling.

\section{Summary}

What was known before

- Acute corneal hydrops is a visually significant complication of keratoconus. It is associated with visual morbidity and increased risk of graft rejection.

- The incidence and management in the UK have not been surveyed previously.

\section{What this study adds}

- This study provided the first estimate of the incidence of acute corneal hydrops in keratoconus It provides additional data for patient counselling.

\section{Conflict of interest}

The authors declare no conflict of interest.

\section{Acknowledgements}

The research was supported by the National Institute for Health Research (NIHR) Biomedical Research Centre based at Moorfields Eye Hospital NHS Foundation Trust and UCL Institute of Ophthalmology.

\section{Disclosure}

The views expressed are those of the authors and not necessarily those of the NHS, the NIHR or the Department of Health.

\section{References}

1 Davidson AE, Hayes S, Hardcastle AJ, Tuft SJ. The pathogenesis of keratoconus. Eye 2014; 28(2): 189-195.

2 Tuft SJ, Moodaley LC, Gregory WM, Davison CR, Buckley RJ. Prognostic factors for the progression of keratoconus. Ophthalmology 1994; 101(3): 439-447.

3 Kelly TL, Williams KA, Coster DJ. Australian Corneal Graft R. Corneal transplantation for keratoconus: a registry study. Arch Ophthalmol 2011; 129(6): 691-697.

4 Pearson AR, Soneji B, Sarvananthan N, Sandford-Smith JH. Does ethnic origin influence the incidence or severity of keratoconus? Eye 2000; 14(Pt 4): 625-628.
5 Kennedy RH, Bourne WM, Dyer JA. A 48-year clinical and epidemiologic study of keratoconus. Am J Ophthalmol 1986; 101(3): 267-273.

6 Georgiou T, Funnell CL, Cassels-Brown A, O'Conor R. Influence of ethnic origin on the incidence of keratoconus and associated atopic disease in Asians and white patients. Eye 2004; 18(4): 379-383.

7 Nielsen K, Hjortdal J, Aagaard Nohr E, Ehlers N. Incidence and prevalence of keratoconus in Denmark. Acta Ophthalmol Scand 2007; 85(8): 890-892.

8 Ihalainen A. Clinical and epidemiological features of keratoconus genetic and external factors in the pathogenesis of the disease. Acta Ophthalmol Suppl 1986; 178: 1-64.

9 Gorskova EN, Sevost'ianov EN. Epidemiology of keratoconus in the Urals. Vestn Oftalmol 1998; 114(4): 38-40.

10 Jonas JB, Nangia V, Matin A, Kulkarni M, Bhojwani K. Prevalence and associations of keratoconus in rural Maharashtra in central India: the central India eye and medical study. Am J Ophthalmol 2009; 148(5): 760-765.

11 Sharma N, Mannan R, Jhanji V, Agarwal T, Pruthi A, Titiyal JS et al. Ultrasound biomicroscopy-guided assessment of acute corneal hydrops. Ophthalmology 2011; 118(11): 2166-2171.

12 Carter JB, Jones DB, Wilhelmus KR. Acute hydrops in pellucid marginal corneal degeneration. Am J Ophthalmol 1989; 107(2): 167-170.

13 Sridhar MS, Mahesh S, Bansal AK, Nutheti R, Rao GN. Pellucid marginal corneal degeneration. Ophthalmology 2004; 111(6): 1102-1107.

14 Fan Gaskin JC, Patel DV, McGhee CN. Acute corneal hydrops in keratoconus-new perspectives. Am J Ophthalmol 2014; 157(5): 921-928.

15 Rowson NJ, Dart JK, Buckley RJ. Corneal neovascularisation in acute hydrops. Eye 1992; 6(Pt 4): 404-406.

16 Donnenfeld ED, Schrier A, Perry HD, Ingraham HJ, Lasonde R, Epstein A et al. Infectious keratitis with corneal perforation associated with corneal hydrops and contact lens wear in keratoconus. $\mathrm{Br}$ J Ophthalmol 1996; 80(5): 409-412.

17 Tuft SJ, Gregory WM, Buckley RJ. Acute corneal hydrops in keratoconus. Ophthalmology 1994; 101(10): 1738-1744.

18 Amsler M. Some data on the problem of keratoconus. Bull Soc Belge Ophtalmol 1961; 129: 331-354.

19 Fan Gaskin JC, Good WR, Jordan CA, Patel DV, McGhee C. The Auckland keratoconus study: identifying predictors of acute corneal hydrops in keratoconus. Clin Exp Optom 2013; 96(2): 208-213.

20 Zadnik K, Barr JT, Gordon MO, Edrington TB. Biomicroscopic signs and disease severity in keratoconus. Collaborative Longitudinal Evaluation of Keratoconus (CLEK) Study Group. Cornea 1996; 15(2): 139-146.

21 Office for National Statistics 2011 Census: Population Estimates for the United Kingdom, 27 March 2011. Available at http://www.ons.gov.uk/ons/rel/census/2011-census/ population-and-household-estimates-for-the-united-kingdom/ stb-2011-census-population-estimates-for-the-united-kingdom. html\#tab-The-structure-of-the-population-of-the-UnitedKingdom.

22 Scotland census 2011. Available at http://www. scotlandscensus.gov.uk/ods-analyser/jsf/tableView/ crosstabTableView.xhtml. accessed on 16 March 2014.

23 Northern Ireland census 2011. Available at http:// www.ninis2.nisra.gov.uk/public/pivotgrid.aspx? dataSetVars=ds-3962-lh-73-yn-2011-sk-136-sn-Census 2011-yearfilter. accessed on 16 March 2014 
24 Kok YO, Tan GF, Loon SC. Review: keratoconus in Asia. Cornea 2012; 31(5): 581-593.

25 Grewal S, Laibson PR, Cohen EJ, Rapuano CJ. Acute hydrops in the corneal ectasias: associated factors and outcomes. Trans Am Ophthalmol Soc 1999; 97: 187-198; discussion 198-203.

26 Panda A, Aggarwal A, Madhavi P, Wagh VB, Dada T, Kumar A et al. Management of acute corneal hydrops secondary to keratoconus with intracameral injection of sulfur hexafluoride (SF6). Cornea 2007; 26(9): 1067-1069.

27 Shah SG, Sridhar MS, Sangwan VS. Acute corneal hydrops treated by intracameral injection of perfluoropropane (C3F8) gas. Am J Ophthalmol 2005; 139(2): 368-370.

28 Feder RS, Wilhelmus KR, Vold SD, O'Grady RB. Intrastromal clefts in keratoconus patients with hydrops. Am J Ophthalmol 1998; 126(1): 9-16.

29 Al Suhaibani AH, Al-Rajhi AA, Al-Motowa S, Wagoner MD Inverse relationship between age and severity and sequelae of acute corneal hydrops associated with keratoconus. Br J Ophthalmol 2007; 91(7): 984-985.

30 Basu S, Vaddavalli PK, Ramappa M, Shah S, Murthy SI, Sangwan VS. Intracameral perfluoropropane gas in the treatment of acute corneal hydrops. Ophthalmology 2011; 118(5): 934-939.

31 Edwards M, Clover GM, Brookes N, Pendergrast D, Chaulk $\mathrm{J}$, McGhee CN. Indications for corneal transplantation in New Zealand: 1991-1999. Cornea 2002; 21(2): 152-155.

32 Anwar HM, Anwar M. Predescemetic dissection for healed hydrops-judicious use of air and fluid. Cornea 2011; 30(12): 1502-1509.

33 Nanavaty MA, Daya SM. Outcomes of deep anterior lamellar keratoplasty in keratoconic eyes with previous hydrops. Br J Ophthalmol 2012; 96(10): 1304-1309.

34 Basu S, Reddy JC, Vaddavalli PK, Vemuganti GK, Sangwan VS. Long-term outcomes of penetrating keratoplasty for keratoconus with resolved corneal hydrops. Cornea 2012; 31(6): 615-620.

35 Statistical Yearbook 2012. Statistics Denmark. Available at http://www.dst.dk/pukora/epub/upload/16251/ 02pop.pdf. accessed on 11 October 2014.

36 Office for National Statistics Ethnicity and National Identity in England and Wales 2011, 11 Dec 2012. Available at http://www.ons.gov.uk/ons/dcp171776_290558.pdf. accessed on 14 March 2014. 